\title{
7 Kommunikative Verfahren und sprachliche Mittel zur Realisierung des Bewertens in Pausengesprächen im Theater
}

In diesem Kapitel sollen zunächst auf Basis erster (Vor-)Analysen (siehe Kapitel 6.5) mit Bezug zur Forschungsliteratur (siehe Kapitel 2) in den untersuchten Gesprächen von den Beteiligten genutzte kommunikative Verfahren und sprachliche Mittel zur Realisierung der Aktivität des Bewertens vorgestellt werden, bevor sie in Kapitel 8 im Zusammenhang mit der Untersuchung sequenzieller Strukturen des interaktiven Vollzugs der Aktivität des Bewertens differenzierter in ihrer sequenziellen Einbettung dargestellt werden.

Die oben genannten (Vor-)Analysen zeigen, dass die Beteiligten in den untersuchten Theaterpausengesprächen Bewertungen unter Zuhilfenahme verschiedenster sprachlicher Mittel realisieren, darunter vor allem lexikalische Bewertungsausdrücke, wie etwa spezifische Adjektive (toll, grauenhaft), Substantive (Glanzleistung) oder auch Verben, die explizit Wertung indizieren (stören, gefallen). Implizite Hinweise auf Bewertungen finden sich - ebenfalls auf der verbalen Ebene - in Form von charakteristischen Modifizierern oder einer wertungstypischen Satzstruktur, Wertung wird in großen Teilen aber auch durch die prosodische Aufladung von Äußerungen vermittelt (siehe Kapitel 8.2 und 8.3). Vor dem Hintergrund dessen, dass das Bewerten von Kunst - in diesem Fall performativer Kunst - auch mit der Demonstration von (Fach-)Wissen, mit Positionierungen und Face-Bedrohungen verbunden ist, lassen sich zudem an der sprachlichen Oberfläche Realisierungen der Beteiligten beobachten, mit dieser potenziellen Gefahr umzugehen. Aufgrund der großen Variationsbreite an kommunikativen Verfahren und sprachlichen Mitteln sind die nachfolgend genannten als eine Auswahl für die in dieser Arbeit untersuchten Gespräche zu betrachten.

\subsection{Bewertungskonstruktionen}

Die Beteiligten realisieren in den dieser Arbeit zugrunde liegenden Gesprächen Bewertungen als objektivierte Bewertungen (Prädikationen) mit Allgemeingültigkeitsanspruch oder als subjektivierte Bewertungen (bewertende Stellungnahmen) mit eingeschränktem Gültigkeitsanspruch. Objektivierte Bewertungen manifestieren sich in den angeführten Ausschnitten in den folgenden Varianten: 


\section{1) BEZUGSOBJEKT ist/war $X$}

\section{Beispiel 12: Köln_14-10-02_Sezuan_Gr.1}

253 Ulrich: aber aber die aKUStik ist furcht [bar-]

Variante 1 besteht in der vollständigen prädikativen Struktur aus in der Regel nominal realisiertem Subjekt, dem Kopulaverb sein und einem undeklinierten, mitunter graduierend modifizierten (Bewertungs-)Adjektiv. Laut Adamzik (1984: 247) ist diese Konstruktion eine wesentliche Äußerungsform von Bewertungen, die, wie Variante 2 zeigt, auch ohne prädikative Verknüpfung durch das Kopulaverb sein realisiert werden kann. Es wird im folgenden Fall zuerst das modifizierte Adjektiv als Wertzuweisung und erst nachfolgend und ohne syntaktische Verknüpfung das Bezugsobjekt genannt.

\section{2) GRADPARTIKEL X BEZUGSOBJEKT}

\section{Beispiel 13: Siegen_15-01-14_7Wellen_Gr.1}

001 Marina: oh VoLL gu: t-

$((.)$.

003 Marina: die inszeNIErung.

In den Varianten 3 und 4 wurde das Bezugsobjekt bereits vom selben oder einem anderen Sprecher in einer (Erst-)Bewertung oder in einer Bewertungseinforderung benannt und wird deshalb in der Folgeäußerung nicht nochmals explizit aufgegriffen (vgl. Hartung 2000: 122).

\section{3) ist/war GRADPARTIKEL/WIDERSPRUCHSMARKER X}

Beispiel 14: Siegen_15-01-14_7Wellen_Gr.2

013 Elfriede: (.) ha ha $h^{\circ}$ (.) war schon-

$014 \quad(0.2)$

015 interesSANT=ne?

4) ist/war GRADPARTIKEL zu X

Beispiel 15: Köln_14-10-02_Sezuan_Gr.1

221 Victoria: für !MICH! war_s natürlich wieder_s $<<$ lachend $>$ gleIche $>$. $<<$ lachend $>$ weisch $>$ ? war BISSL- 
Die evaluative lexikalische Einheit X kann durch eine Gradpartikel intensiviert oder abgeschwächt werden, ihr kann auch die Abtönungspartikel schon als Widerspruchsmarker (Variante 3) vorangehen, um Distanz zur Vorgängeräußerung zu indizieren oder ihr den Status einer nicht übereinstimmenden Bewertung zuzuschreiben. Das einem beschreibenden Adjektiv vorangestellte Adverb $z u$ ist Ausdruck einer mit Blick auf ein implizites Ziel unzweckmäßig hohen Ausprägung einer Eigenschaft (vgl. Melis 2014) und indiziert in den in dieser Arbeit untersuchten Beispielen eine negative Wertung (Variante 4).

Subjektivierte Bewertungen stellen die zweite Realisierungsmöglichkeit dar. Die in dieser Arbeit präsentierten Beispiele zeigen, dass subjektivierte, das heißt in ihrem Gültigkeitsanspruch auf den Sprecher begrenzte Bewertungen häufiger realisiert werden als objektivierte Bewertungen, das heißt solche mit Allgemeingültigkeitsanspruch. Da eine subjektivierte Bewertung „kennzeichnet, daß die ganze Person beteiligt ist“, kann diese mit Fiehler (2014: 49) auch als bewertende Stellungnahme bezeichnet werden (im Gegensatz zu objektivierten Bewertungen, die zumindest durch ihre sprachliche Form keine explizite persönliche Involviertheit indizieren). Nach Finegan (1995: 1) bringt der Sprecher mit einer subjektivierten Äußerung sein Selbst zum Ausdruck und macht seine Perspektive und Haltung im Gespräch deutlich (,speaker's imprint“) (siehe auch Kapitel 2.3.4). Sprachliche Realisierungen subjektivierter Bewertungen zeigen in den in dieser Arbeit analysierten Gesprächsausschnitten das im Folgenden dargestellte Varianzspektrum.

Mit der Einbettung unter finden als Verb der propositionalen Einstellung in der 1. Person Singular, im Präsens (ich finde) oder im Präteritum (ich fand), nimmt der Sprecher explizit auf sich in seiner „befindenden“ beziehungsweise wertenden Haltung sprachlich Bezug. Auf diese Weise wird die Gültigkeit der unter dem Verb eingebetteten wertenden Prädikation, die über ein Objektsprädikativ wie in Variante 5 oder in Form einer satzwertigen Prädikation wie in Variante 6 realisiert sein kann, prophylaktisch (vgl. Stein 1995: 202) als einzig aus Sicht des Sprechers gültig eingeschränkt (vgl. König 2014: 217 sowie auch Imo 2012).

5) ich finde/fand BEZUGSOBJEKT $X$

Beispiel 16: Köln_14-04-22_BrainAndBeauty_Gr.3

448 Emma: ((schmatzt)) ich fand den PERser gut.

449 der in dem (. ) in dem DUNk[elblauen kosTüM. ] 
6) ich finde/fand BEZUGSOBJEKT ist/war $\mathrm{X}$

\section{Beispiel 17: Köln_14-10-02_Sezuan_Gr.1}

232 Ulrich: ich find vor ALlem so diese-

234

die kuLISsen sind originell;

Auch in der folgenden Variante 7 wird die Bewertung durch eine Subjektivierung in ihrer Gültigkeit eingeschränkt. Das subjektivierende find ich, hier im Präsens, steht allerdings nicht wie in den vorherigen Varianten äußerungsinitial, sondern äußerungsintern im Anschluss an die Nennung des Bezugsobjekts.

\section{7) BEZUGSOBJEKT finde/fand ich GRADPARTIKEL $X$}

Beispiel 18: Siegen_14-11-14_Hamlet_Gr.2

396 Moritz: d]ie SCHAUspielerin find ich auch brutal397 FÜRCHterlich?

In Variante 8 wird die Bewertung ebenfalls durch ein äußerungsinternes fand ich subjektiviert. Das Bezugsobjekt der Bewertung wird bereits durch das der Subjektivierung vorangestellte Korrelat das projiziert und erst durch einen mit dass an die Bewertung angeschlossenen Objektsatz konkretisiert.

8) das finde/fand ich $X$ dass BEZUGSOBJEKT $X$ war

Beispiel 19: Köln_14-07-03_DieLücke_Gr.2

192 Vanessa: aber das fand [ich] EH ganz gut dass die so ähm$((. .)$.

198 Vanessa: dass es so AUFklärend war ne,

Eine nachträgliche Abschwächung einer vorerst objektivierten Bewertung in Form einer Prädikation tritt in Variante 9 auf. Hier werden die Subjektivierung der Äußerung und die damit verbundene Relativierung des Gültigkeitsanspruchs der Bewertung erst äußerungsfinal vorgenommen.

9) BEZUGSOBJEKT ist/war GRADPARTIKEL $X$ finde/fand ich

Beispiel 20: Köln_14-10-02_Sezuan_Gr.1

319 Ulrich: [und die] !GÖT!ter sind_n bissel diletTANtisch

fInd ich. 
In Variante 10 wird die Bewertung von einem äußerungsinitialen ich finds sowie einem äußerungsfinalen glaub ich gerahmt. Die äußerungsinitiale Subjektivierung indiziert, dass die Bewertung lediglich für den Sprecher gilt. Durch die äußerungsfinale und unbetonte Subjektivierung glaub ich rahmt der Sprecher seine Äußerung, die - obwohl in ihrem Gültigkeitsanspruch explizit auf sein individuelles Empfinden begrenzt - ,aus irgendeinem Grund problematisch sein könnte (Verständnisprobleme der Gesprächspartner, mögliche Gesichtsbedrohung, Unsicherheit über den Wahrheitsgehalt der Äußerung etc.)“ als unsicher (Imo 2006: 277).

10) ich finde/fand BEZUGSOBJEKT GRADPARTIKEL zu X glaube ich

\section{Beispiel 21: Köln_14-07-03_DieLücke_Gr.2}

092 Vanessa: ich finds $n$ bisschen ZU: ANgreifend glaub ich an die andern aber-

Die angeführten Varianten zeigen, dass die an den Bewertungsinteraktionen beteiligten Sprecher ihre Bewertungen subjektivieren, um so deren Gültigkeitsanspruch beziehungsweise Gewissheit (vgl. Deppermann 2015) einzuschränken und als allein für sich zutreffend markieren. Indem sie durch eine Subjektivierung, die auch nachträglich vorgenommen werden kann (glaubich), die mit ihrer Bewertung vorgenommene Positionierung (epistemic stance, siehe Kapitel 2.3.3) als unsicher und damit als heikel und potenziell Face-bedrohend markieren sowie die Gültigkeit der Bewertung einschränken, bewahren sich die Beteiligten nach Stein (1995: 202) Rückzugsmöglichkeiten: „Werden Äußerungen als partiell unzutreffend entlarvt oder vollständig in Frage gestellt oder sogar widerlegt“, so Stein (1995: 202), „trägt eine derartige Markierung von Äußerung(steil)en dazu bei, sich vor einem Imageverlust zu schützen“.

\subsection{Lexikalische Mittel}

$\mathrm{Zu}$ den lexikalischen Mitteln, die von den Beteiligten in den vorliegenden Gesprächen genutzt werden, um Bewertungen zu realisieren, zählen Adjektive, Substantive, Verben, Phrasen/Formeln und solche Mittel, die sprachliche Vagheit indizieren, beispielsweise Hedging, ${ }^{81}$ vage Verweise, Verweise auf den Wissensstatus und Disclaimer. ${ }^{82}$ Diese Mittel werden im Folgenden anhand von Beispielen erläutert.

$81 \mathrm{Zu}$ einer Erläuterung des Begriffs ,Hedging، siehe Kapitel 7.3.

$82 \mathrm{Zu}$ einer Erläuterung des Begriffs, ,Disclaimer‘ siehe ebenfalls Kapitel 7.3. 


\subsubsection{Adjektive}

Die These, dass Adjektive typischerweise zur Realisierung von Bewertungen herangezogen werden (vgl. Adamzik 1984: 247), wird auch durch die Ergebnisse, die aus den Analysen in der vorliegenden Arbeit hervorgegangen sind, gestützt, denn in den untersuchten Gesprächen machen Adjektive den größten Anteil an Bewertungsausdrücken aus. Zudem werden sie in vielen Fällen durch skalierende Adverbien oder adverbiale Adjektive wie ziemlich, ganz, voll, richtig, wirklich, unglaublich, sehr, echt, brutal, super oder wahnsinnig intensiviert oder abgeschwächt und weisen zum Teil einen emotionalen Bedeutungsanteil auf (vgl. Sandig 1979: 142). Die Bewertungsinteraktionen zeigen eine große Bandbreite an Adjektiven, wie zum Beispiel klasse, toll, super, originell oder schön, die von den Beteiligten zur positiven Evaluation genutzt werden, sowie an Adjektiven wie schlecht, grauenhaft, furchtbar, hässlich oder billig, die der negativen Evaluation dienen. Adjektive wie verwirrend, interessant oder krass lassen sowohl eine positive als auch negative Lesart zu, indem sie lediglich ein Bedeutungsmoment des Auffälligen beinhalten. Sie treten in den vorliegenden Gesprächen oftmals in Erstbewertungen auf und dienen den Beteiligten aufgrund ihrer offenen Lesart möglicherweise als Vorsichtsmaßnahme, ihre Bewertung und die damit verbundene Positionierung weniger angreifbar zu machen beziehungsweise den Zugzwang zu umgehen, sich eindeutig positionieren zu müssen. Ob diese Bewertungsausdrücke negativ oder positiv zu deuten sind, wird gegebenenfalls an der Art und Weise ihrer paraverbalen Realisierung und durch ihre emotionale beziehungsweise evaluative Aufladung deutlich. In diesem Fall wäre die Lesart vorgegeben und die Funktion als Vorsichtmaßnahme nicht gegeben. Wird die Lesart allerdings nicht paraverbal indiziert, so bleibt die (positive oder negative) Ausrichtung der Bewertung vage und wird möglicherweise in der Folgeäußerung des Gegenübers konkretisiert. Wertung wird auch durch „extreme und emotional engagierte BEWERTUNGEN“ (Adamzik 1984: 257, Herv. im Original) mithilfe von „Extremwörtern“ (Adamzik 1984: 255), das heißt Adjektiven wie beschissen oder spitze, sowie durch Superlative (in attributiver Stellung) (am besten, schlechteste) vorgenommen. Laut Marschall (2011: 98) sind bewertende Adjektive nicht als „geschlossenes, klar umrissenes Feld“ abgrenzbar, sondern einer relativ geringen Anzahl an rein evaluativen Adjektiven steht eine Vielzahl an Adjektiven gegenüber, „bei denen die Bewertung als Teilbedeutung auf einer beschreibenden Leistung beruht [. . . ] und durch diese gerechtfertigt wird“. In diesem Fall wird in den in dieser Arbeit analysierten Gesprächen die bewertende Teilbedeutung oftmals durch eine paraverbale Markierung betont (siehe Kapitel 8.2 und 8.3). 


\subsubsection{Substantive}

Neben wertenden Adjektiven können auch wertende Substantive als sprachliche Mittel des Bewertens eingesetzt werden. Hier überwiegen nach Adamzik (1984: 249) vor allem „Wörter, bei denen die Wertung nur zur konnotativen Bedeutung gehört“. Wertende Substantive treten in den in dieser Arbeit analysierten Gesprächen hauptsächlich im Rahmen von negativen Bewertungen auf. Die Substantive sind allesamt Komposita und erhalten ihre Wertung durch einen im vorliegenden Kontext bewertenden Bedeutungsanteil und eine affektive Aufladung auf der paraverbalen Ebene. In den folgenden Beispielen verwendet Moritz die Substantive Schultheaterniveau, Abziehbilder und Schultheaterrequisitenbüro zur negativen Bewertung der von ihm besuchten Inszenierung und als JargonAusdrücke, um sich als Kunstkenner zu positionieren.

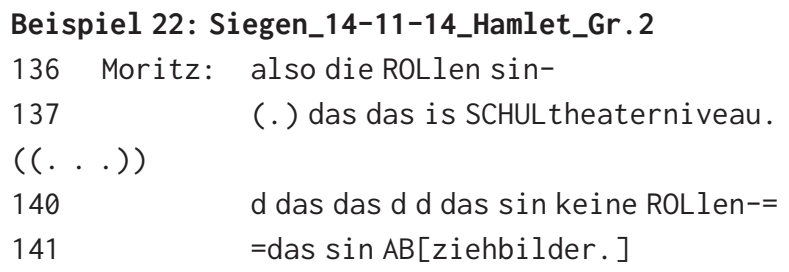

Ulrich bezeichnet in Beispiel 24 im Stück agierende Figuren als Azubigötter, nachdem er diese bereits vorangehend mit dem Adjektiv dilettantisch negativ bewertet hat (siehe Kapitel 7.1).

Beispiel 24: Köln_14-10-02_Sezuan_Gr.1

323 Ulrich: die WIRke wie so:-

$((.)$.

326 aZUbigötter-

Marina hingegen bewertet in Beispiel 25 die schauspielerische Performance der im Stück Agierenden mit dem Substantiv Glanzleistung positiv. 


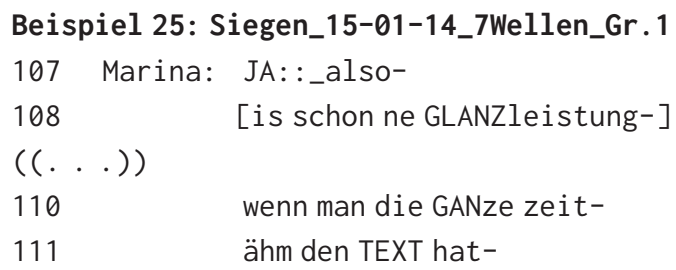

Ähnlich wie die zur Intensivierung eingesetzten Skalierer können auch Artikel eine intensivierende Funktion übernehmen. Diese Funktion ist bei dem Demonstrativpronomen diese $/ r / s$ in attributiver Stellung gegeben: Es kann den Beteiligten dazu dienen, das nachfolgende Substantiv beziehungsweise die nachfolgende Nominalgruppe „exaltiert heraus[zu]stellen und so jeweils den Kern-/Bezugspunkt ihrer Aussage und insbesondere ihrer (häufig vor allem emotiven) Bewertung (und damit ihr jeweiliges Bezugsobjekt) [zu] betonen“ (Hrncal/Gerwinski 2015: 55) und sich - vor allem bei negativen Bewertungen - vom Bezugsobjekt zu distanzieren.

\subsubsection{Verben}

Ebenso wie Substantive treten auch Verben zur Bewertung selten auf. In den Pausengesprächen dienen Verben wie langweilen, (jmd.) anmachen (hier im Sinne von diskriminieren), rumfuchteln, schreien, stören, mögen oder gefallen den Beteiligten als Mittel zur Indikation einer negativen oder positiven Bewertung und zur Verbalisierung positiver oder negativer Eindrücke (vgl. Adamzik 1984: 251). Im Falle von langweilen, mögen, gefallen oder verwirren indizieren Verben „einen emotionalen Zustand, den das Objekt im Sprecher hervorruft“ (Adamzik 1984: 251). Demgegenüber beschreiben anmachen, rumfuchteln und schreien nicht das Empfinden der Zuschauer als Rezipienten, sondern das rezipierte Geschehen, schließen dabei aber zusätzlich eine Sprecherwertung ein.

\subsubsection{Phrasen/Formeln}

Neben einzelnen lexikalischen Elementen finden sich in den in dieser Arbeit untersuchten Gesprächen auch Wertung indizierende Phrasen, darunter verbale wie vom Hocker gehauen, auf die Nerven fallen oder mit dem Holzhammer daherkommen sowie adjektivische wie over (the) top. 


\subsection{Sprachliche Vagheit}

Die Analysen der in dieser Arbeit angeführten Gesprächsausschnitte zeigen auch, dass die Beteiligten in großen Teilen auf sprachliche Mittel zur Abschwächung von Bewertungen zurückgreifen, um diese als heikel, weniger verbindlich und unsicher zu markieren und so das mit einer Bewertung verbundene Problem der Face-Bedrohung (vgl. Brown/Levinson 1987: 271-272) durch defensives FaceWork (vgl. Goffmann 1967) zu lösen. Obwohl die zugehörigen Mittel nicht selbst wertend sind, sind sie doch Teil der Praxis des Bewertens.

\subsubsection{Hedging}

Als Hedges bezeichnet Lakoff (1973: 471) „words whose job is to make things fuzzier or less fuzzy“. Nach Clemen (1997: 241) tritt Hedging in Form sprachlicher Vagheit typischerweise in solchen Kommunikationssituationen hervor, in denen Äußerungen nicht präzise evaluiert werden können. Hedges werden von den Sprechern genutzt, um ihre Einstellung hinsichtlich ihrer Äußerung beziehungsweise Positionierung zum Ausdruck zu bringen (vgl. Clemen 1997: 244). Außerdem können Sprecher mithilfe von Hedging unter anderem bereits eine potenzielle Nichtübereinstimmung des Gesprächspartners projizieren (vgl. Clemen 1997: 244) beziehungsweise sich im Vorhinein gegen diese absichern. In der vorliegenden Arbeit verlieren die Bewertungen beispielsweise durch Heckenausdrücke (Hedges) wie ein bisschen, ein(en) Ticken, eigentlich, irgendwie, wahrscheinlich an Verbindlichkeit (vgl. auch Adamzik 1984: 255). Indem die an den Bewertungsinteraktionen Beteiligten ihre Bewertungen durch Hedging als unscharf markieren, können sie indizieren, dass ihre durch die Bewertung vorgenommenen Posititionierungen potenziell heikel und Face-bedrohend sind und sich, wie auch durch die explizite Subjektivierung, weniger angreifbar machen. Elemente des Hedging treten sowohl in selbstinitiierten als auch in eingeforderten Bewertungen, in positiven und negativen (Erst-)Bewertungen sowie bei Nichtübereinstimmung auf.

\subsubsection{Vage Verweise}

In den analysierten Gesprächsausschnitten ist zu beobachten, dass von den Beteiligten teilweise nur vage (zumeist mit einem deiktischen Verweis durch die Proform es) auf das Bezugsobjekt ihrer Bewertung verwiesen wird (vgl. auch Baldauf 2001). Konkretisierungen werden nicht notwendigerweise eingefordert, erst 
nachträglich geliefert oder im Rahmen einer Zweitbewertung vom Gegenüber vorgenommen.

Im folgenden Beispiel erfragt Ina von Finn eine bewertende Stellungnahme des nicht näher von ihr konkretisierten Bezugsobjekts es:

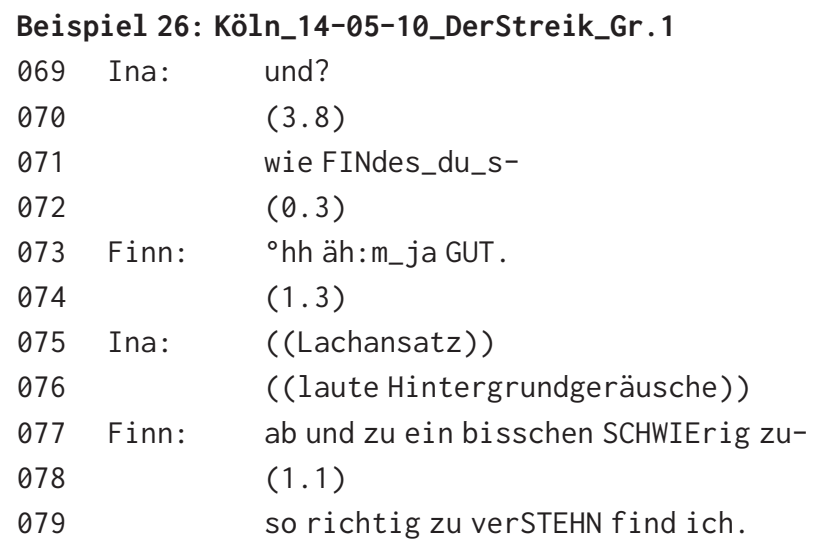

Finn liefert die eingeforderte Stellungnahme, in der er das Bezugsobjekt vorerst noch nicht konkretisiert. Die Konkretisierung nimmt er in einem zweiten Schritt nach einer Pause mit dem Verweis auf Verständnisprobleme vor. Dass die Konkretisierung des Bezugsobjekts der Bewertung durch den Gesprächspartner vorgenommen werden kann, zeigt sich auch im folgenden Beispiel:

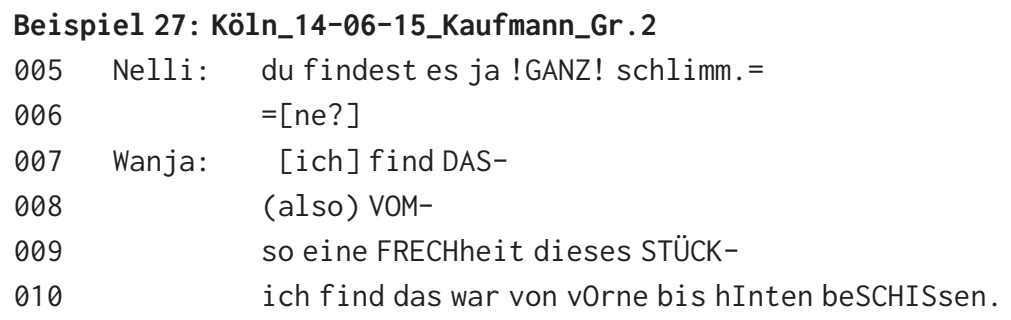

Während Nelli bei ihrer Bewertungsunterstellung vage mit der Proform es auf das Bezugsobjekt verweist, konkretisiert Wanja dieses, indem er den Fokus auf das Theaterstück legt und es negativ bewertet. Auch im nächsten Beispiel hält Titus das Bezugsobjekt der von Vanessa eingeforderten Stellungnahme mit der Proform es vage:

Beispiel 28: Köln_14-07-03_DieLücke_Gr.2

005 Vanessa: und? 
006

007 Titus: ja es [war schon KRASS also hehehe]

Im folgenden Beispiel verweist Donata mittels des Demonstrativpronomens „diese“, mit dem sie gleichzeitig gemeinsames Hintergrundwissen unterstellt, und mittels des Verweises „so_n paar“ auf im Stück agierende Schauspieler, die sie positiv bewertet:

\section{Beispiel 29: Köln_14-04-22_BrainAndBeauty_Gr.1}

013 Donata: ich hätt ja gern noch EINzelapplaus.

014 geHABT.

$015 \quad(0.9)$

$016 \quad$ für diese einzelnen DARsteller?

$017 \quad$ =da warn ja so_n paar wirklich S[Uper ]=ne?

018 Viktoria: [ja_JA.]

Viktoria nimmt weder eine Konkretisierung des Bezugsobjekts vor, noch fordert sie eine Konkretisierung ein, sondern stimmt ihrer Gesprächspartnerin zu. Im Gegensatz zu den vorangehenden Beispielen erfolgt die Konkretisierung im folgenden Beispiel nicht selbst-, sondern fremdinitiiert:

Beispiel 30: Köln_14-07-03_DieLücke_Gr.2

043 Titus: es GAB so n paar stelln da hab ich echt gedacht-

044

$(0.7)$

045

046

047 Vanessa: muss das jetz SEIN,

048 Titus: ja (.) wo der richtig LAUT geworden is;

049 richtig LAUT geschrien hat so-

Auf Titus’ vagen Verweis „so n paar stelln“ fordert Vanessa mit ihrer Nachfrage explizit eine Konkretisierung des Bezugsobjekts von Titus ein, die er im Anschluss auch liefert.

\subsubsection{Verweise auf den Wissensstatus}

Dass mit Bewertungen auch (implizit) der Wissensstatus der Beteiligten und damit verbundene Bewertungsrechte ausgehandelt werden, wurde in Kapitel 2.3.3 bereits 
aufgezeigt. Explizite Verweise auf Nichtwissen (,epistemische Unschärfemarkierungen“, König 2014: 184) wie weiß nicht oder keine Ahnung werden von den Beteiligten in den vorliegenden Gesprächen genutzt, um mit ihren Bewertungen beanspruchte epistemische Rechte abzuschwächen, Bewertungen als begründungsbedürftig zu behandeln, zu indizieren, dass ihnen Beispiele zur Untermauerung ihrer Begründungen oder Bewertungen aktuell nicht zugänglich sind, oder sie fungieren als Disfluenzmarker bei Formulierungsschwierigkeiten (vgl. auch König 2014). Unter Disfluenzmarkern verstehe ich mit Fischer (1992: 1) Elemente wie beispielsweise „Schweigepausen, semantisch inhaltsleere Partikeln, Vokalund Konsonantendehnungen“ sowie „verschiedene Formen der Selbstkorrektur“, die den Beteiligten im Gespräch als Ressourcen zur Verzögerung und Kontextualisierung (vgl. Auer 1986; Schmitt 1993) ihrer Äußerungen dienen.

Titus versucht im folgenden Beispiel zu begründen, warum er einige Stellen im Theaterstück negativ bewertet. Auf die Aufforderung seiner Gesprächspartnerin Vanessa hin konkretisiert er zuerst die Stellen, um die es geht, und liefert dann Gründe für seine Bewertung:

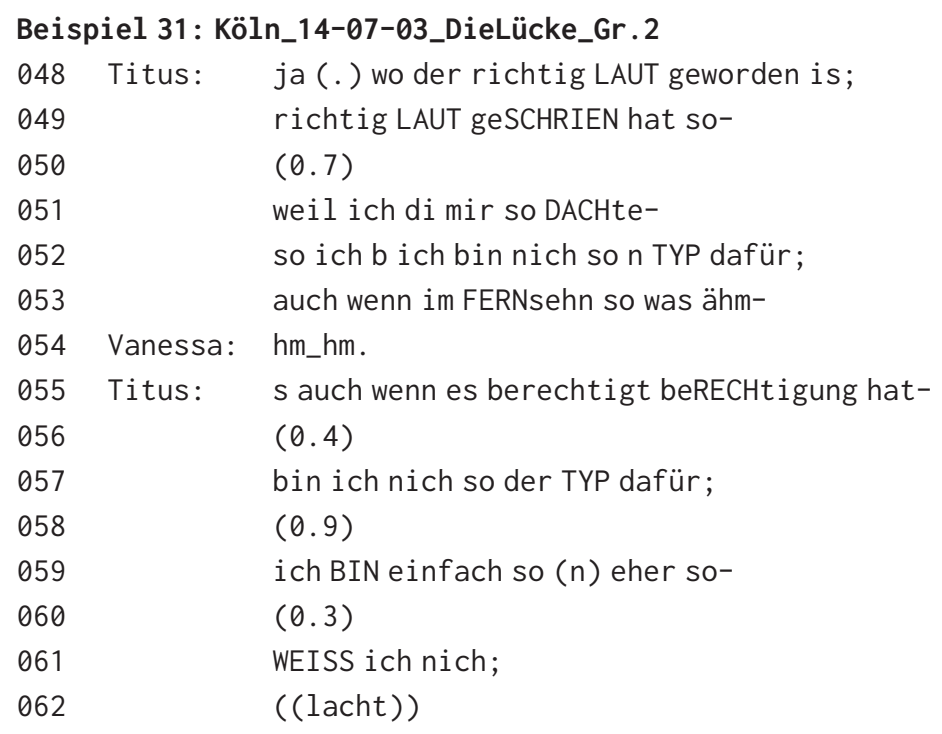

Seine Bewertung versucht Titus durch Selbstzuschreibungen beziehungsweise Selbstkategorisierungen zu begründen, die er allerdings mit „WEISS ich nich“ abbricht und damit indiziert, dass ihm weitere Beschreibungen aktuell nicht zugänglich sind. Auch im nächsten Beispiel fungiert „ich WEISS nich“ als epistemische Unschärfemarkierung: 


\section{Beispiel 32: Siegen_14-11-14_Hamlet_Gr.2}

229 Moritz: s[o:-a ]lso weder irgendwie beGRÜNdet noch irgendwie-

$230 \quad(0.2)$

231 also es_is ECHT so so ! HM! .=

232 =jetz machen wir malsch $\mathrm{n}$ bisschen theAter-=

233 =machen ändern jetzt das LICHT-

234

235

236

${ }^{\circ} \mathrm{h}$ das licht reagiert übrigens auch zu SPÄT-

ALso?

237

238

239

es-

240

$(0.7)$

241

ich WEISS nich-

242

wahrscheinlich ham sie nen andern TECHniker?

$(0.3)$

Wie auch Titus im vorangehenden Beispiel bricht Moritz in diesem Beispiel die Formulierung der Begründung seiner Bewertung mit dem Verweis darauf ab, dass ihm weitere Gründe oder Beschreibungen möglicherweise nicht zugänglich sind. Dass Moritz im Gegensatz zu Titus, der nach seiner epistemischen Unschärfemarkierung die Bewertungs- und Begründungssequenz nicht weiter expandiert und mit einem Lachen als abgeschlossen rahmt, eine Vermutung als weiteren Grund anführt, weist darauf hin, dass „ich WEISS nicht“ hier zudem die Funktion eines Disfluenzmarkers übernimmt und somit auch Formulierungsarbeit indizieren kann (vgl. König 2014: 187).

Dass die Unschärfemarkierung keine Ahnung ähnlich wie der explizite Verweis auf Nichtwissen in den vorangehenden Beispielen eingesetzt werden kann, zeigt das nächste Beispiel:

\section{Beispiel 33: Siegen_14-10-26_Blut_Gr.2}

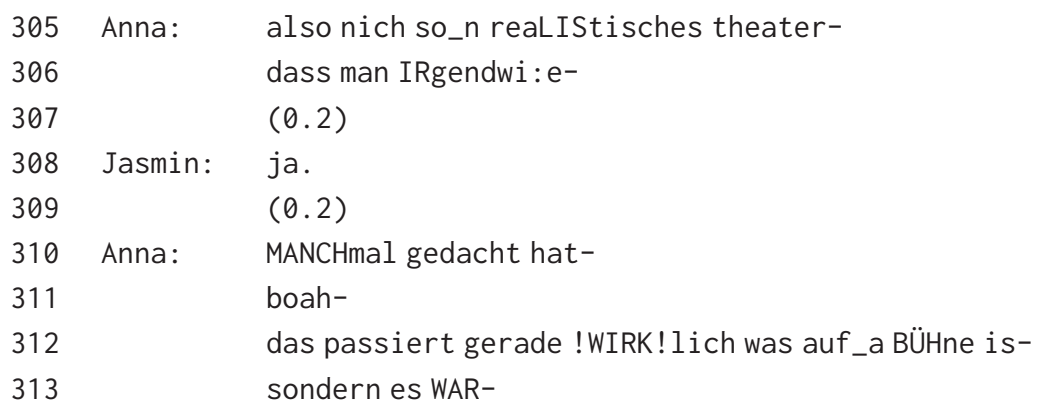




$\begin{array}{ll}314 & { }^{\circ} \text { S SCHON so- } \\ 315 & \text { dass man die ganze zeit WUSSte-= } \\ 316 & =\text { oKE :- } \\ 317 & \text { es is_n theAterstück- } \\ 318 & \text { und so WEIter- } \\ 319 & \text { die sind da in ihren ROLlen und so- } \\ 320 & { }^{\circ} \text { h also (.) keine AHnung. } \\ 321 & =\text { ich hab auch mal theAterstücke gesehen- } \\ 322 & \text { WO das- } \\ 323 & \text { oh !SO! rea! LIS! tisch geSPIELT wurde- } \\ 324 & \text { dass du irgendwie VOLL- } \\ 325 & (0.8)\end{array}$

Anna versucht ihre Bewertung des Theaterstücks durch einen Vergleich mit einem anderen Theaterstück, dass sie bereits gesehen hat, zu begründen. Dass sie den Vergleich sehr schnell an ihre epistemische Unschärfemarkierung „keine AHnung “ anschließt, spricht dafür, dass sie in diesem Fall als Disfluenzmarker im Rahmen von Formulierungsarbeit dient.

Im Folgenden wird „KEIne ahnung“ von Pascal im Zuge einer Deutungssequenz eingesetzt, die sich an eine Bewertung anschließt:

\section{Beispiel 34: Siegen_14-10-26_Blut_Gr.2}

$\begin{array}{lll}445 & \text { Pascal: } & \text { ALso au die auch die !GANZ! am ANfang- } \\ 446 & \text { Anna: } & \text { [JA ja: } \\ 447 & \text { Pascal: } & \text { [die doch EIgentlich] dann [in diese: } r-] \\ 448 & \text { Anna: } & \\ 449 & \text { Pascal: } & \text { Ebene waren- } \\ 450 & & \text { [JA_ja: } \\ 451 & & \text { KE sie AUCH schon nur ge (.) das (.) } x \times x- \\ 452 & & \text { (das) ENde war verwirrend. } \\ 453 & & (0.2)\end{array}$

Pascal indiziert durch den Verweis „KEIne ahnung“ in diesem Beispiel Schwierigkeiten bei der Deutung des im Theaterstück Rezipierten sowie dass ihm aktuell keine weiteren Deutungsalternativen zugänglich sind.

Auch die bereits in Kapitel 7.1 angesprochenen Rahmungen der Bewertungen durch eine äußerungsfinale Ergänzung des Verbs glauben in der 1. Person Singular Präsens dient neben der Subjektivierung und damit der Einschränkung der beanspruchten Gültigkeit der vorgenommenen Positionierungen zugleich einem 
impliziten Verweis auf den Wissensstatus beziehungsweise Wissenszugang der betreffenden Sprecher (vgl. Imo 2012; siehe auch Kapitel 2.3.3).

\subsubsection{Disclaimer}

Bruder und Ucok (2000: 342) haben basierend auf Untersuchungen der Kommunikation vor Kunstwerken im Museum herausgestellt, dass Disclaimer als sprachliches Mittel bei Bewertungen im Kontext der Kunstkommunikation eine wichtige Rolle spielen und von den Beteiligten eingesetzt werden, um sich von ihrem eigenen Urteil zu distanzieren (siehe Kapitel 3.1 sowie Stoltenburg 2009: 273). Auch in den dieser Arbeit zugrunde liegenden Pausengesprächen treten Disclaimer als möglicher Bestandteil von Bewertungen und als sprachliches Mittel zur Abschwächung auf.

Im Gespräch zwischen Gabriele und Cecilia stellt Cecilia ihrer von Gabriele eingeforderten Bewertung den Disclaimer „EHRlich gesagt“ voran:

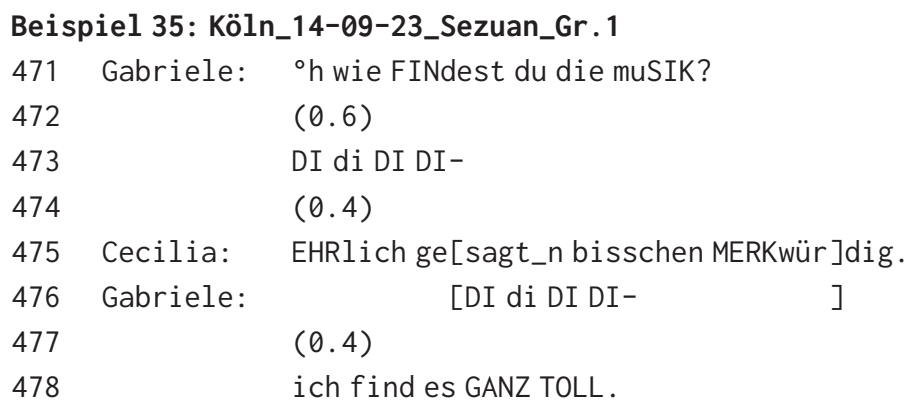

Mit dem ihrer Bewertung vorangestellten Disclaimer markiert Cecilia ihre Bewertung und die damit vorgenommene Positionierung als potenziell heikel, und macht sich weniger angreifbar. „EHRlich gesagt“ könnte Cecilia hier möglicherweise auch dazu dienen, sich von der mit ihrer Bewertung beanspruchten Verantwortung/Position zu distanzieren (vgl. auch Stoltenburg 2009)

Die Funktion der Abschwächung erfüllt auch der Disclaimer ,also !ICH! persönlich“ im folgenden Beispiel:

Beispiel 36: Siegen_14-11-14_Hamlet_Gr.2

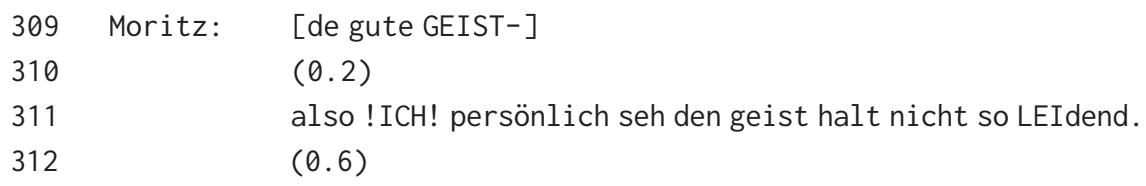


Indem Moritz seiner Bewertung den Disclaimer voranstellt, markiert er seine anschließende implizit negative Bewertung als explizit subjektiv und schränkt somit die mit der Bewertung beanspruchte Gültigkeit prophylaktisch ein (vgl. Stein 1995: 202), um seine Positionierung, wie Cecilia im vorangehenden Beispiel, weniger angreifbar zu machen. Diese Funktion ähnelt dem in Kapitel 7.1 vorgestellten Verfahren der äußerungsinitialen Subjektivierung. Geht man mit Hewitt und Stokes (1975: 3) davon aus, dass Disclaimer sprachliche Mittel sind, um im Vorfeld (der Äußerung) negative Zuschreibungen durch das Gegenüber abzuwenden, die die Äußerung beziehungsweise das Betragen (conduct) des Sprechers möglicherweise zur Folge haben könnte, dann könnten teilweise auch den in Kapitel 8 dargestellten Subjektivierungen Funktionen eines Disclaimers zugesprochen werden.

\subsection{Implizite Mittel}

Im Falle von impliziten Bewertungen greifen die Beteiligten nicht auf Wörter mit evaluativem Bedeutungsanteil (vgl. Keller 2008: 14) zurück, sondern nutzen verstärkt paraverbale Merkmale zur Indikation von Wertung. Ein weiteres Verfahren zur Indikation von Wertung ist nach Keller (2008: 14) die Realisierung von Bewertungen in Form verbaler Äußerungen ohne explizit evaluative Elemente, die im vorliegenden Kontext aber dennoch auf eine Bewertung schließen lassen und denen auch von den Beteiligten der Status einer Bewertung zugeschrieben wird. Diese Realisierung von Bewertungen im „pragmatischen Modus“ (vgl. Keller 2008: 14) setzt allerdings Kontextwissen und -sensitivität voraus und birgt deshalb auch die Gefahr, dass eine implizite Bewertung nicht als solche von den Beteiligten behandelt wird. Trifft diese Beschreibung Kellers für schriftliche Kommunikate zu, so muss sie im Hinblick auf die in der vorliegenden Arbeit analysierten Gespräche relativiert werden: Auch wenn die Äußerung keine explizit evaluativen Elemente enthält, trägt sie eine prosodische Konturierung, die es den Beteiligten ermöglicht, die Äußerung als Bewertung zu interpretieren (,assessment signals“, Goodwin/Goodwin 1987: 7-8), was beispielsweise im Falle rein schriftlicher Kommunikation nicht der Fall ist.

In den Gesprächen auftretende implizite verbale Bewertungen werden beispielsweise durch die Äußerung, sich das Theaterstück nicht weiter ansehen zu wollen, realisiert:

Beispiel 37: Siegen_14-11-14_Hamlet_Gr.2

331 Moritz: ich fand_s ICH o (ich) also ganz ehrlich.

332

(0.4) 
Auch der Verweis auf das aktuelle körperliche Befinden kann einer impliziten Bewertung dienen:

Beispiel 38: Siegen_14-11-14_Hamlet_Gr.2

101 Moritz: ich habe seit etwa (. ) geFÜHLT DREI stunden $<<$ lachend $>$ ! KOPF! schmerzen $>$.

102 $(0.6)$

$103 \quad$ WEgen diesem stü-

104 $h^{\circ}$ DURCH dieses stück.

Eine weitere Variante implizieter Bewertungen ist das Äußern in der Aufführung nicht eingelöster Wünsche oder Erwartungen:

\section{Beispiel 39: Köln_14-04-22_BrainAndBeauty_Gr.1}

013 Donata: ich hätt ja gern noch EINzelapplaus.

014 geHABT.

$015 \quad(0.9)$

016 für diese einzelnen DARsteller?

017 =da warn ja so_n paar wirklich S[Uper]=ne?

Auch „erlebensdeklarative“ Verbalisierungen (vgl. Fiehler 2014: 45) wie beispielsweise Titus’ Äußerung im folgenden Ausschnitt dienen der Indikation von Wertung:

Beispiel 40: Köln_14-07-03_DieLücke_Gr.2

043 Titus: es GAB so $n$ paar STELLN da hab ich echt gedacht-

$044 \quad(0.7)$

045 muss das jetz SEIN,

\subsubsection{Paraverbale Aufladung von Beschreibungen}

Die bereits in Kapitel 2.1.3 angesprochene Schwierigkeit der Differenzierung zwischen Bewertungen und Beschreibungen können sich die Beteiligten zunutze machen, indem sie eher deskriptive Ausdrücke äußern und diese paraverbal mit 
Wertung aufladen (vgl. dazu auch Uhmann 1996), wie dies etwa im Gespräch zwischen Gudrun und Susanne der Fall ist:

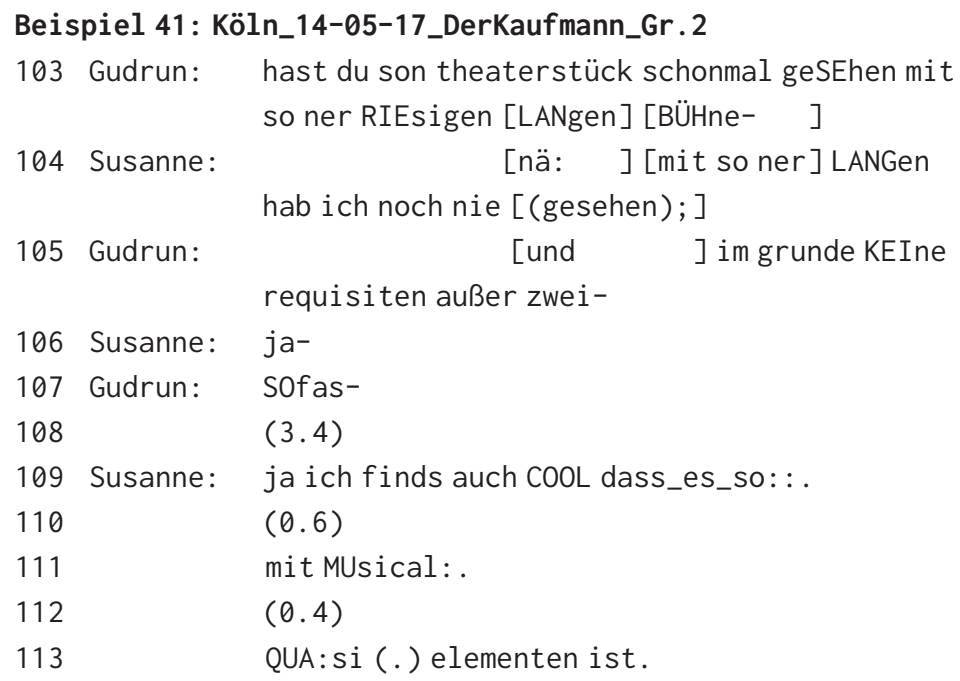

In diesem Beispiel führt die prosodische Realisierung eigentlich beschreibender Ausführungen zur Bühne und zum Bühnenbild zu einer Bewertung von Susanne, die darauf hinweist, dass sie die beschreibenden Äußerungen von Gudrun bereits als Bewertung interpretiert.

Eine weitere Möglichkeit, die Schwierigkeit der Abgrenzung von Beschreibungen und Bewertungen zu nutzen, besteht in der Möglichkeit, eine sachliche Aussage zu verbalisieren, „die aufgrund ihres außersprachlichen Kontextes, allgemeiner oder spezieller Wissensvoraussetzungen als wertend zu interpretieren ist“" (Adamzik 1984: 255). Dies ist im folgenden Ausschnitt der Fall, in dem Finn Verständnisprobleme äußert. Dass Ina dieser Wahrnehmungsbeschreibung Finns im vorliegenden Kontext den Status einer Bewertung zuschreibt, wird an der Form ihrer Äußerung (Zustimmung + deklarative Bewertung + Einladung zur Bestätigung durch ein Rückversicherungssignal) deutlich:

\section{Beispiel 42: Köln_14-05-10_DerStreik_Gr}

077 Finn: ab und zu ein bisschen SCHWIErig zu-

$078 \quad(1.1)$

079 so richtig zu verSTEHN find ich.

$080 \quad(0.3)$

081 al[so: . ]

082 Ina: [ja das] is sehr TEXTlastigne? 
Der dritten Möglichkeit zur Realisierung einer Bewertung ohne explizite lexikalische evaluative Elemente bedient sich Victoria im folgenden Ausschnitt:

Beispiel 43: Köln_14-10-02_Sezuan_Gr.1

221 Victoria: für !MICH! war_s natürlich wieder_s $<<$ lachend $>$ glEIche $>$.

$222 \quad<<$ lachend $>$ weisch $>$ ?

223 war BISSL-

$224 \quad(0.2)$

225 zu LANG-

Indem Victoria dem beschreibenden Adjektiv „LANG“ die Gradpartikel „zu“ voranstellt, schreibt sie dem Bezugsobjekt ihrer Bewertung (in diesem Fall dem Theaterstück) „eine von der Wertnorm negativ abweichende Eigenschaft“ zu (Adamzik 1984: 255), ohne allerdings die Wertnorm zu explizieren; dass es sich jedoch um eine subjektive handelt, wird an der Phrase für mich deutlich, die funktional einem Dativus Iudicantis entspricht.

\subsubsection{Sprechen mit fremder Stimme}

Ein in den Gesprächen zu beobachtendes zentrales Verfahren des Indizierens von Wertung ist das paraverbale, affektive Aufladen von Äußerungen (vgl. auch Sandig 1979: 142). Dies geschieht vor allem im Rahmen des Sprechens mit fremder Stimme aus der Perspektive einer anderen Person. Das Sprechen mit fremder Stimme dient den Beteiligten in den analysierten Gesprächsausschnitten der parodierenden Reinszenierung derjenigen, deren Perspektive eingenommen wird (vgl. auch Baldauf 2001: 81 sowie Kapitel 3.1). Durch diesen systematischen Wechsel (vgl. Meyer/Meier zu Verl 2013: 223) in die Rolle einer anderen Person und das Sprechen aus deren Perspektive, begleitet von einer paraverbal affektiven Aufladung der Äußerungen, werden vor allem negative Bewertungen implizit realisiert. Dieses Verfahren zur Indizierung von negativer Kritik nutzt Dina im folgenden Beispiel bei der Darstellung ihrer Englischlehrerin, um sich von deren Aussage zu distanzieren:

Beispiel 44: Köln_14-09-25_DieLücke_Gr.3

177 Dina: mirist-

$178 \quad(0.5)$

179 also- $\mathrm{h}^{\circ}$

$180 \quad(0.2)$ 
Auch Moritz nutzt in den folgenden Ausschnitten sehr extensiv das Sprechen mit fremder Stimme in Kombination mit einer affektiven Aufladung, um die Personen, aus deren Perspektive er spricht, und ihr Vorgehen zu kritisieren:

\section{Beispiel 45: Siegen_14-11-14_Hamlet_Gr.2}

118 Moritz: <<mit tiefer Stimme imitierend> jetz mach ich theAter.

119 ha_HA-

120 (. ) there shall you PASS-

121 (. ) you shall not PASS wa[_a>. ]

Beispiel 46: Siegen_14-11-14_Hamlet_Gr.2

152 Moritz: guck do mal wie die leute reaGIEren.

153 s_is so <<imitierend> ! OH! mein STICHwort kam.

154 ich komm jetz mal auf die BÜHne.

155 !OH! mein STICHwort kam.

156 ich muss jetz die HAND geben.

157 !OH! mein STICHwort kam.

158 ich muss auf den BOden gucken>.

Beispiel 47: Siegen_14-11-14_Hamlet_Gr.2

224 Moritz: ${ }^{\circ}$ un dann auf einmal gibt es diesen LICHTswitchund <<imitierend $>$ ! HO! jetzt bin ich auf der MEtaebene>.

228 Marco: [ja_JA:-]

229 Moritz: s[o:-a ]lso weder irgendwie beGRÜNdet noch irgendwie-

$230 \quad(0.2)$

Beispiel 48: Siegen_14-11-14_Hamlet_Gr.2

481 Moritz: [und WIE lockern wir_s] auf- 
${ }^{\circ} \mathrm{h}<<$ mit verstellter Stimme $>$ indem wir bisschen dumm RUM $>$ -

483 $(0.7)$ $<<$ mit verstellter Stimme> HIhihi.

486 Marco: ${ }^{\circ}$ h ui [UIo>. ]

487 Moritz: öhm um rum äh äh öh LAUtö: generieren.

Auch Wanja spricht im folgenden Beispiel mit fremder Stimme, um das Agieren der Figuren im Stück zu bewerten:

\section{Beispiel 49: Köln_14-06-15_Kaufmann_Gr.2}

218 Wanja: das trägt_shalt !ü!berHAUPT nich-

219 diese SINgerei dazwischen?

220 $(0.6)$

$221 \quad$ !KOM! plett UNmotiviert-

222 ja:?

$223(0.5)$

224 und JETZT komma nomma dran (ü)ben-

225 (die/hier) MÜSST ich ne: ä:h-

226 ne LEIter falschrum aufstellen.

Während Moritz und Wanja in den vorangehenden Beispielen das Sprechen mit fremder Stimme dazu dient, Figuren im Stück zu bewerten, nutzt Ulrich das Sprechen aus der Rolle der im Stück agierenden Götter als Ressource zur Vervollständigung seiner zuvor abgebrochenen, beschreibenden Äußerung in Zeile 330:

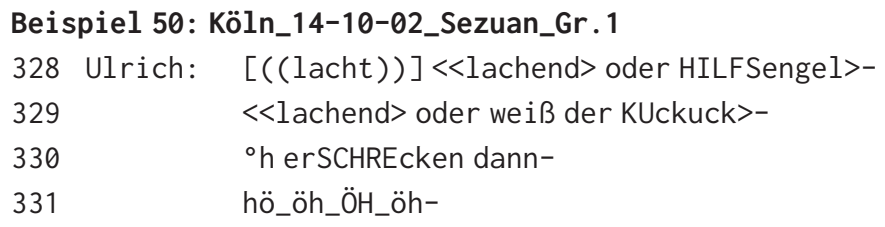

Der Wechsel in die Rolle einer anderen Person wird in den angeführten Beispielen anhand der prosodischen Realisierung in einem betont affektierten Sprachmodus deutlich, der von der paraverbalen Realisierung der unmittelbar vorangehenden Äußerung abweicht und so einen Bruch in der Intonationskontur hervorruft. Diese Parodisierungen sprechen nach Klemm (2001) zum einen dafür, dass der Aspekt des Sich-Vergnügens bei den Bewertungsinteraktionen auch eine Rolle spielt, zum anderen können sie einer psychischen Entlastung des Sprechers dienen, die in dieser Form während der Rezeption des Theaterstücks nicht möglich ist. 


\subsection{Formen der Begründung von Bewertungen}

Die explizite Darlegung von Wertmaßstäben und die Argumentation unter Rückgriff auf (fachliches) Hintergrundwissen werden von den Beteiligten selten als Begründung ihrer Bewertungen angeführt. Stattdessen begründen sie ihre Bewertung durch Belehrungen (um etwa bei Nichtübereinstimmung ihr Gegenüber doch noch zur Zustimmung zu bewegen), durch Erlebensdeklarationen, indem sie das emotionale Erleben oder ihre Wahrnehmungen während der Rezeption des Theaterstücks schildern, durch das Anführen von Beispielen aus dem Theaterstück, durch die Beschreibung situativer Umstände einer Erfahrung, durch Projektionen und Szenarien („,was wäre, wenn ... “), durch Vergleiche und Kontrastierungen mit anderen Theaterstücken, oder durch Bekundungen persönlicher Vorlieben.

\subsubsection{Belehrungen}

Bereits in Kapitel 2.3.2 wurde auf den Face-bedrohenden Charakter nicht explizit eingeforderter Belehrungen hingewiesen. Diese Face-Bedrohung liegt darin begründet, das Belehrungen nach Keppler (1989: 539) zunächst ein „doppeltes Ungleichgewicht" herstellen, das sich zum einen in einer Wissensasymmetrie (Keppler spricht von einem epistemischen Gefälle) und zum anderen in einem „kommunikative[n] Gefälle“ zwischen den Beteiligten niederschlägt (vgl. auch Kotthoff 1995: 59). Keppler (1989: 541) unterscheidet auf Basis ihrer Analyse privater Alltagsgespräche des Weiteren zwei Arten von Belehrungen, darunter als häufigste Form solche, die durch Nachfragen von einer anderen Person initiiert werden. ${ }^{83}$ Bei selbstinitiierten, das heißt nicht explizit durch eine andere Person eingeforderten Belehrungen muss sich der Belehrende hingegen zunächst „in einer Art Vorspann“ (Keppler 1989: 546, Herv. im Original) das Rederecht von den anderen Beteiligten einholen, sodass er seiner selbstinitiierten Belehrung dadurch den Charakter einer eingeforderten Belehrung verleiht. Dieses Vorgehen macht deutlich, dass Belehrungen, die nicht fremdinitiiert sind - zumindest in (privaten) Alltagsinteraktionen - eine dispräferierte Variante darstellen (vgl. Keppler 1989: 546) und die Belehrenden zudem Gefahr laufen, den bisherigen Wissensstatus der belehrten Person nicht adäquat einzuschätzen. Belehrungen können entweder zu einem Ausgleich einer vorliegenden

83 Dass sich die Häufigkeit des Vorkommens eingeforderter und selbstinitiierter Belehrungen institutionsspezifisch unterscheiden kann, das heißt von den Beteiligten gegebenenfalls öfter auf die eigentlich dispräferierte Variante selbstinitiierter Belehrungen zurückgegriffen wird, zeigt zum Beispiel Weidner (2017) bei der Analyse von Kochsendungen im Fernsehen. 
Wissensasymmetrie führen oder diese weiter verstärken (vgl. Kotthoff 1995: 58). Hat der Belehrende seine Belehrung abgeschlossen, so stehen dem Belehrten verschiedene Möglichkeiten zur Verfügung, um zu ratifizieren, dass eine Wissensvermittlung stattgefunden hat beziehungsweise die für die Belehrung ausschlaggebende Wissensasymmetrie behoben wurde (vgl. Keppler 1989: 551-553).

Belehrungen in den vorliegenden Gesprächen beziehen sich primär auf die Vermittlung von mit den rezpierten Theaterstücken zusammenhängendem Sachund Fachwissen und werden von den Beteiligten genutzt, um Bewertungen zu untermauern und durch Wissensasymmetrie begründete Nichtübereinstimmung so möglicherweise aufzuheben. In Beispiel 51 verleiht Cecilia durch die paraverbale Markierung des Adjektivs „SCHRÄG“ ihrer Äußerung eine negative Wertung. Gabriele indiziert mit dem von ihr geäußerten Widerspruchsmarker „JA aber“, bestehend aus der Antwortpartikel ja und der adversativen Konjunktion aber, Nichtübereinstimmung und klärt Cecilia dann darüber auf, dass bei Brecht fast alle Gesänge schräg seien:

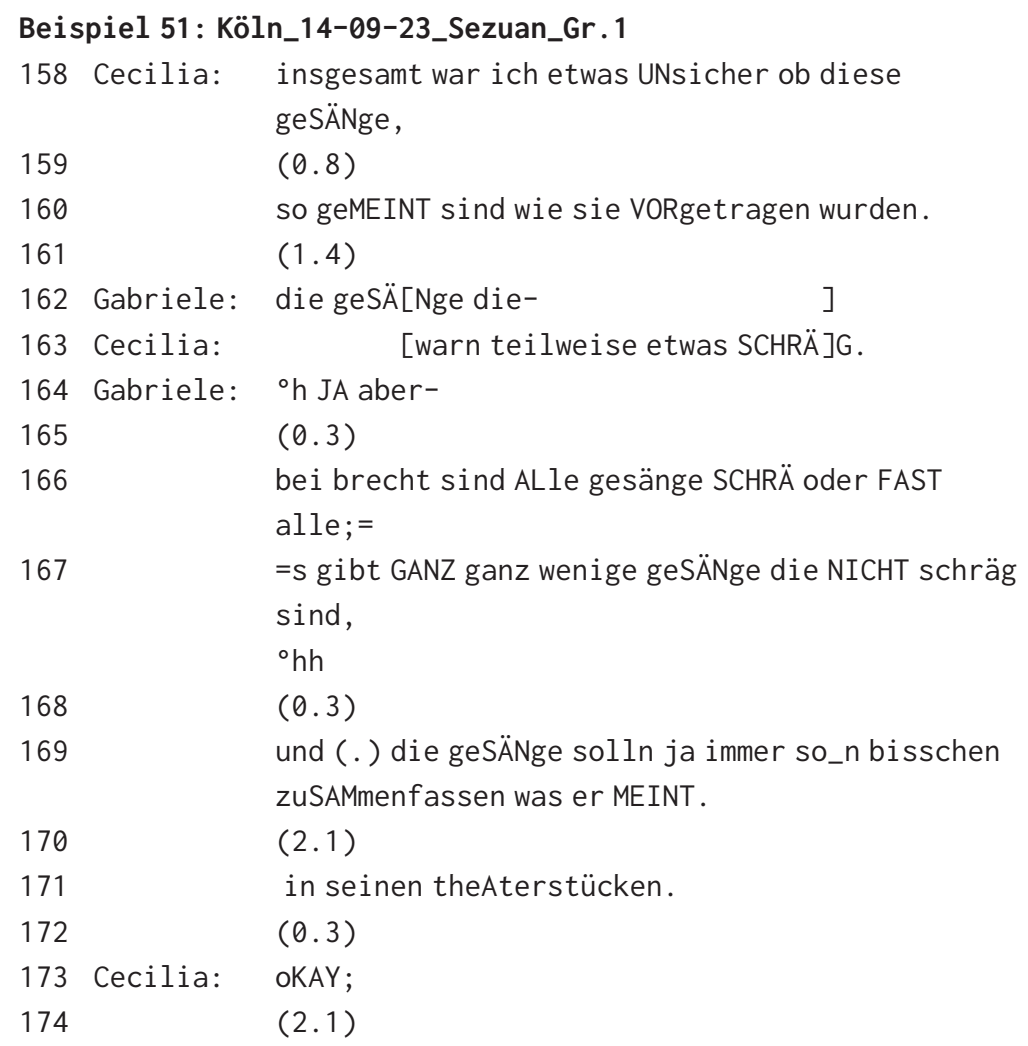


Im Rahmen ihrer Belehrung behebt Gabriele nicht nur die zwischen ihr und Cecilia bestehende Wissensasymmetrie (vgl. Luckmann 1989: 41; Günthner/Knoblauch 1994: 700-701), sondern unterstellt mittels ihres Verweises „die geSÄNge solln ja immer so_n bisschen zuSAMmenfassen was er MEINT“ (Z. 169) zudem durch die Partikel „ja“ gemeinsames Hintergrundwissen. Außerdem ist die selbstinitiierte Belehrung Gabrieles ein Indiz dafür, dass sie Cecilias verbalisierte Unsicherheit, wie die Gesänge im Stück zu verstehen sind, als implizite Einladung zur Wissensvermittlung und damit auch zu einer Belehrung deutet.

\subsubsection{Erlebensdeklarationen}

Im Zuge von Begründungen durch Erlebensdeklarationen (vgl. Fiehler 2014: 45) legen die Beteiligten ihr emotionales Erleben sowie ihre Wahrnehmungen dar, verbalisieren gegebenenfalls Denk- und Deutungsprozesse, machen diese so den anderen Gesprächsteilnehmern zugänglich und somit einen gemeinsamen Abgleich des Erlebten und Wahrgenommenen möglich (siehe Kapitel 8). Charakteristische Mittel zur Realisierung von Erlebensdeklarationen sind mentale Prozessverben (zum Beispiel überlegen, denken).

In Beispiel 52 verbalisiert Anna ihren Denkprozess während der Rezeption des Theaterstücks und macht diesen somit den anderen am Gespräch Beteiligten zugänglich und die Gründe für ihre Bewertung transparenter:

\section{Beispiel 52: Siegen_14-10-26_Blut_Gr.2}

248 Anna: =also es WAR ja dann letztendlich ! AUCH! irgendwie $n$ the! $\mathrm{A}$ ! ter. $=$ =aber das fand ich voll interesSANT. ${ }^{\circ} \mathrm{h}$ am ANfang hab ich gedachtoKE :zwischendurch schalten die LICHter um.

255 =wann SCHALten denn die lichter eigentlich um:-

256 und DANN war_s halt irgendwie so-

257 ja je nachdem wo die geRAde-

258 in welcher ROLle die gerade irgendwie stecken.

Vanessa begründet ihre negative Bewertung im folgenden Ausschnitt ebenfalls durch das Verbalisieren ihrer Gedanken: 


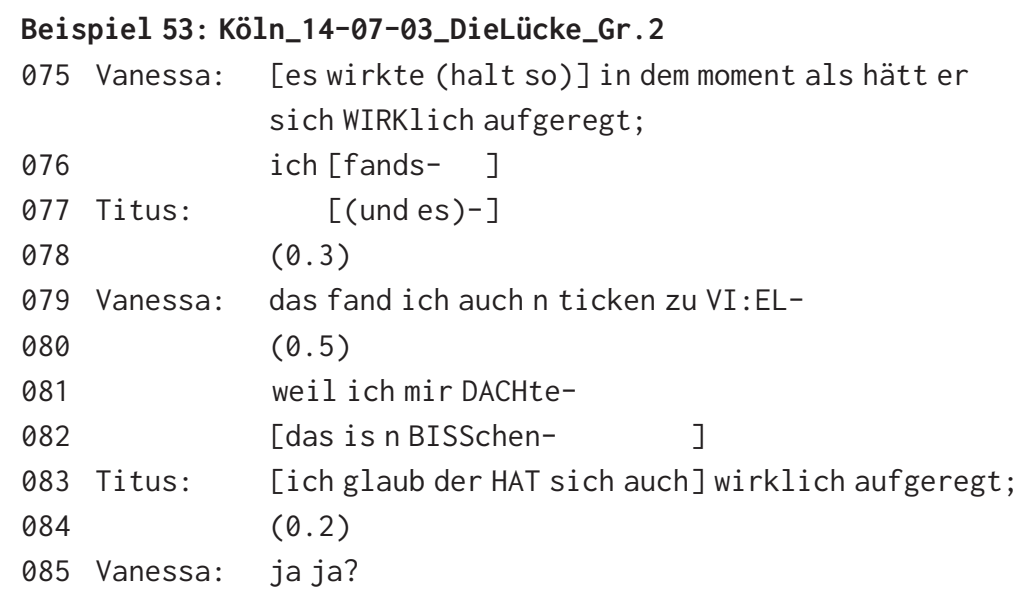

Mit der Rekonstruktion ihres Denkprozesses lässt Vanessa Titus an ihrer Rezeptionserfahrung teilhaben und ermöglicht einen Abgleich der Wahrnehmungen, an dem sich Titus auch beteiligt, indem er in Überlappung mit Vanessas Äußerung ihre Wahrnehmung bestätigt.

\subsubsection{Erfahrungsbeschreibungen und Moralisierungen}

In Erfahrungsbeschreibungen werden die „situativen Umstände, in deren Rahmen ein Erlebnis stattfand“, geschildert (Fiehler 2014: 44). Mithilfe von Erfahrungsbeschreibungen stützen die Beteiligten ihre Bewertungen, indem sie eigene Erfahrungen schildern, dann häufig in Episoden, die in der 1. Person und in einem Vergangenheitstempus realisiert sind, und diese mit den im Stück präsentierten Inhalten vergleichen. Die Schilderung eines konkreten Falls beziehungsweise der Abgleich von selbst erlebten Ereignissen mit der Darstellung gleicher oder ähnlicher Ereignisse im Theaterstück unterstützt die Sprecher dabei, ihr Gegenüber von ihrer Bewertung zu überzeugen (vgl. Günthner/Knoblauch 1994: 700) und diese $\mathrm{zu}$ begründen. Außerdem bieten Erfahrungsbeschreibungen die Möglichkeit für einen Übertrag des Rezipierten auf die gesellschaftliche Ebene außerhalb des Theaterstücks und ermöglichen Moralisierungen.

Titus begründet in Beispiel 54 seine negative Bewertung einiger Stellen im Stück durch die Schilderung persönlicher Erfahrungen:

Beispiel 54: Köln_14-07-03_DieLücke_Gr.2

$\begin{array}{ll}219 \text { Titus: } & \text { also ähm- } \\ 220 & (1.1)\end{array}$


221

222

223

224

225

226

227

228

229

230

231

232

233 Vanessa:

234

235 Titus:

236

237

238

239

240

241 Vanessa:

242 Titus:

243

244

245

246

247

248

249

250

251

252

253

254

255 also ich fands au wie gesacht auch gut KRItisch

(. ) gemAcht-

$(0.9)$

ähm-

(0.3)

es gab so $n$ paar STELLN wo ich ähm-

(2.1)

dem ich vielleicht nich so ZUstimmen würde;

(0.3)

wo es um die reliGIOnen ging und so was;

weil ich hatte da auch schon mal EIgene

erfahrungen gemacht-

(1.0)

[ähm- ]

[mit WAS] denn?

$(0.7)$

ä: h m ich äh HATte ja auch-

ich war in einer beRUFSschule,

(0.8)

und dann sollte: $\mathrm{n}$ medchen ZWANGSverheiratet werden;

(0.2)

und wir hatten uns drum geKÜMmert;

das hast du glaub ich ma erZ̈̈HLT;

das hatt ich aber schonma erZ̈̈HLT;

$(0.5)$

also-

$(0.3)$

damit will ich nur SAgen dass ähm-

(0.8)

es GIBT dinge die äh-

$(0.5)$

die passiern halt SCHON;

(0.4)

und da muss man SCHON irgendwie gucken dass es irgendwie-

(0.7)

dass man da auch HILFT;

und dass man da auch irgendwie in irgendeiner FORM ähm- 
256

257

258
(1.0)

nich SAGT-

äh das geht mich nichts AN;

Im Anschluss an seine Erfahrungsbeschreibung verbalisiert Titus explizit (Z. 246), dass ihm die Beschreibung einer persönlichen Erfahrung dazu dient, seine an die Erfahrungsbeschreibung angeschlossene Moralisierung zu rechtfertigen.

\subsubsection{Beispiele}

Zur Begründung von Bewertungen führen die Beteiligten unter anderem auch Inhalte aus dem Theaterstück als Beispiele an. Dies ist im folgenden Ausschnitt aus dem Gespräch zwischen Nelli und Wanja der Fall:

\section{Beispiel 55: Köln_14-06-15_Kaufmann_Gr.2}

290 Wanja: was ham die denn zur STOry beigetragen.

291 das war dann einfach nur so_n PAUsenclown.

$292 \quad(0.3)$

293 Nelli: nee: .

294 das STIMMT nicht.

295 die be HAM ja-

296 die waren ja HANDlungsträger.

$297 \quad(0.3)$

298

299

die ham ja ZWIschendurch immer-

300

301 Wanja:

beRICHtet-

302

dit [was jetz zum BEI] spiel ähm:-

Nelli:

[hhh $^{\circ}$

]

304

305

(0.3)

306 Unbekannt: was MEINST du.

307 Nelli: das war ja SCHON so a:ls ä:h-

308

309

310

is das jetz hier zu LAUT?

311

312

$\mathrm{hh}^{\circ}$

313

das war ja SCHON: - 


$\begin{array}{ll}314 & (0.6) \\ 315 & \text { so dass die SAchen: ä: hm:- } \\ 316 & \text { erZ̈̈HLT haben- } \\ 317 & \text { die im HINtergrund pasSIERT sind auch- } \\ 318 & (0.6) \\ 319 & \text { ALso:- } \\ 320 & (0.2) \\ 321 \text { Wanja: } & \text { Aber- } \\ 322 & (5.7) \\ 323 & ((\text { stellt Tasse ab)) wenigstens is der } \\ & \text { KAFfee gut. } \\ 324 & (0.3)\end{array}$

Nelli versucht durch das Rekonstruieren einer Stelle im Theaterstück Wanjas negative Bewertung zweier im Stück dargestellter Figuren zu widerlegen, ihre konträre Position zu rechtfertigen und Wanja durch das von ihr angeführte Beispiel doch noch von ihrer positiven Bewertung der beiden Figuren zu überzeugen, was ihr allerdings nicht gelingt.

\subsubsection{Projektionen}

Im folgenden Ausschnitt wird die negative Bewertung der im Stück präsentierten Lehrerfigur durch eine Projektion begründet. Nach Annalenas impliziter Bewertung der Figur projizieren die vier Gesprächsteilnehmer diese in die gesellschaftliche Wirklichkeit außerhalb des Theaterstücks und konstruieren ein Szenario, das ausdrucksseitig durch den Konjuktiv II (Irrealis) markiert ist („was wäre, wenn“).

Beispiel 56: Siegen_14-10-26_Blut_Gr.2

388 Annalena: JA:_a.

389 das war AUCH dieses:-

390 stereoTYpisch<<vibrierend $>$ e: $>$.

$391 \quad(($ lacht $))$ unfähige $<<$ lachend $>$ LEHrerbild $>$ ?

392 Anna: ja: .

$393 \quad(0.4)$

394 Pascal: mh-

395 Annalena: AH_oh.

$396 \quad(0.5)$

397 mh_JOA. 


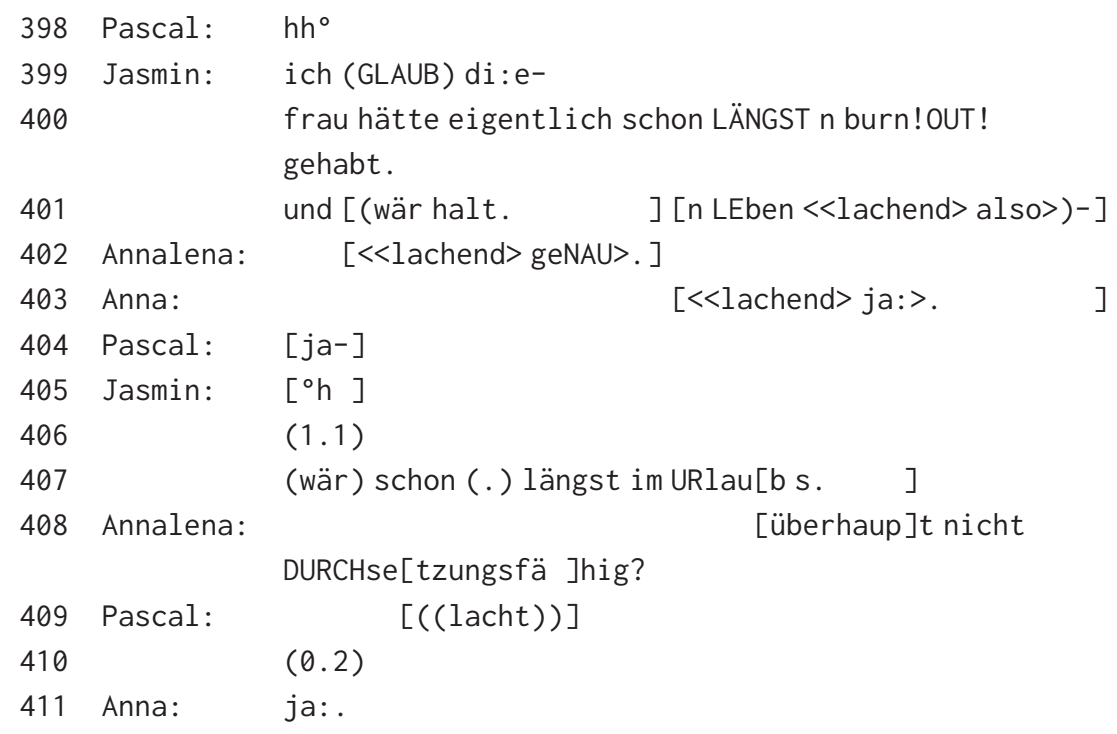

Der Ausschnitt zeigt, dass das Begründen einer Bewertung keine individuelle Leistung des Bewertenden ist, sondern dass Begründungen von allen am Gespräch beteiligten Personen realisiert und ausgehandelt werden können.

\subsubsection{Vergleiche und Kontrastierungen}

Um eine positive oder negative Bewertung zu rechtfertigen, greifen die Beteiligten auch auf Vergleiche der besuchten Inszenierung mit anderen Theaterstücken zurück oder stellen das Rezipierte und andere bereits besuchte Stücke in einen Kontrast zueinander. Im folgenden Ausschnitt führt Anna - im Rahmen der gemeinsamen Begründung der positiven Bewertung des Bühnenbilds einer anderen Gesprächspartnerin - andere bereits von ihr besuchte Theaterstücke und ihre Rezeptionserfahrungen an und kontrastiert diese mit dem aktuell rezipierten Theaterstück:

Beispiel 57: Siegen_14-10-26_Blut_Gr.2

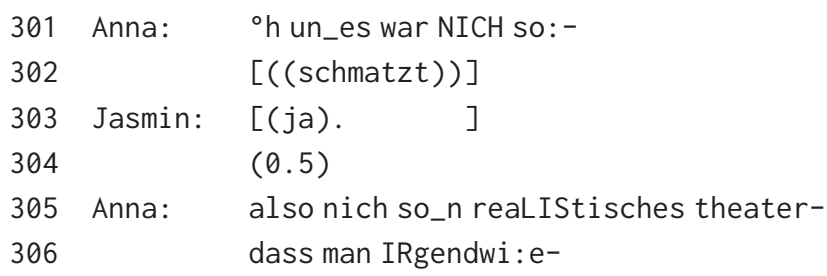


307

308 Jasmin: ja.

309

310 Anna:

311

312

313

314

315

316

317

318

319

320

321

322

323

324

325

326

327

328

329

330

331

332

333

334
(0.2)

$(0.2)$

MANCHmal gedacht hat-

boah-

das passiert gerade !WIRK! lich was auf_a BÜHne issondern es WAR-

${ }^{\circ} \mathrm{h}$ SCHON So-

dass man die ganze zeit WUSSte-=

=oKE : -

es is_n theAterstück-

und so WEIter-

die sind da in ihren ROLlen und so-

${ }^{\circ} \mathrm{h}$ also (.) keine AHnung.

=ich hab auch mal theAterstücke gesehen-

wo das-

${ }^{\circ} \mathrm{h}$ ! SO! rea! LIS! tisch geSPIELT wurde-

dass du irgendwie VOLL-

(0.8)

also RICHtig da !DRIN! warst.=

=als wenn das gerade !WIRK! lich pasSIEren würde.=

=und NICHT nur irgendwie so_n the! A! terstück wär.

' $h$ aber das fand ich da ja NICH. =

=also alLEIN schon durch diese-

${ }^{\circ}$ hh ä:h-

(0.4)

tz $\ddot{\mathrm{A}}: \mathrm{H}-$

LIEder da zwischendurch und so-

Im nächsten Ausschnitt führt Holger den Vergleich zu anderen an der gleichen Spielstätte rezipierten Inszenierungen an, um seine Bewertung zu rechtfertigen:

\section{Beispiel 58: Köln_14-05-24_DerKaufmann_Gr.1}

128 Holger: [also] das äh-

129 Adelheid: ja?

130 Holger: BÜHnenbild find ich hier SUper klasse.

131 Adelheid: ach $\uparrow E C H T \uparrow ?$

132 Holger: du $\uparrow$ NICH$\uparrow ?$

133 Adelheid: (eh pff) also SUper KLASse find ich jetzt en BISSchen überTRIEben aber äh- 
134 Holger: also für das was ich [SONST so] hier schon geSEhen

135 Adelheid: [ja? ]

136 Holger: ${ }_{1}$ [habe. ich find]

137 Adelheid: 1 [ach SO? ]

138 Holger: ${ }_{2}$ [das sehr sehr toll]

139 Adelheid: ${ }_{2}$ [ok im verGLEICH. ]

140 Holger: ${ }_{3}$ [mit DIEser- ]

141 Adelheid: ${ }_{3}[$ ich finde das $]$ AUCH gut ja.

Durch den impliziten Vergleich, den Adelheid in Zeile 139 explizit als Vergleich benennt, gelingt es Holger, Adelheid doch noch von seiner Bewertung des Bühnenbilds zu überzeugen.

\subsubsection{Geschmacksbekundungen}

Ein weiteres Verfahren der Begründung von Bewertungen ist das Äußern von Geschmacksbekundungen, die vor allem durch das Modalverb mögen indiziert werden.

So führt Dina im folgenden Beispiel persönliche Geschmacksvorlieben als implizite Begründung ihrer positiven Bewertung des Bühnenbilds an:

Beispiel 59: Köln_14-09-25_DieLücke_Gr.3

311 Susanne: aber des mit dem BÜHnenbild des find ich schön dass dir_s gefällt. =

312 =ich find_s nämlich AUCH klasse.

$313 \quad(1.0)$

314 Dina: ich mag das GERne.

$315 \quad$ ich mag das SEHR gerne.

316 wenn das (. ) BÜHnenbild irgendwie auch so_n BISSchen-

$317 \quad(0.4)$

318 moderniTÄT zeigt.

319 weißte wenn des NICH so_n.

$320 \quad(1.4)$

$321 \quad$ KONservatives bühnenbild is das man irgendwie erwArten KÖNNte.

Auch Marco begründet im folgenden Ausschnitt seine extrem formulierte positive Bewertung durch das Anführen persönlicher Geschmacksvorlieben: 
Beispiel 60: Siegen_14-11-14_Hamlet_Gr.2

123 Moritz: so UNglaublich pathetisch-

$124 \quad{ }^{\circ} \mathrm{h}$ sie KÖNN-

125 Marco: find ich aber total GEIL-

126 weil i ich auf das BRItische stehe.

127 ${ }^{\circ} \mathrm{h}(($ lacht $))$

Die in diesem Kapitel dargestellte Auswahl zeigt, dass den Beteiligten ein breites Spektrum an kommunikativen Verfahren und sprachlichen Mitteln zur Realisierung des Bewertens zur Verfügung steht. Die angeführten Verfahren und Mittel verdeutlichten, dass Bewertungen nicht nur mittels explizit evaluierender Ausdrücke realisiert werden können, sondern dass sich den Beteiligten viele Möglichkeiten bieten, Bewertungen und ihre Begründungen auch implizit zu kommunizieren. Wie sie die mit dem Bewerten im Kontext der Kunstkommunikation verbundene Face-Bedrohung sowie das Management nichtübereinstimmender Bewertungen bewältigen, wird ebenso anhand der vorangehend angeführten Auswahl deutlich. Im folgenden Kapitel 8 geht es nun darum aufzuzeigen, wie die oben dargestellten kommunikativen Verfahren und sprachlichen Mittel von den Beteiligten in den dieser Arbeit zugrunde liegenden Gesprächen sequenziell eingebettet werden, um das Bewerten als „unfolding process“ (Goodwin 2018: 151) in unterschiedlichen Ausprägungsvarianten sequenziell $\mathrm{zu}$ realisieren. 